Elizabeth Miramontes Moreno 


\section{Argumentos en favor de la teoría de la explicación causal en filosofía de la acción}

\section{Introducción}

En el presente escrito pretendo analizar las acciones humanas; partiendo de la pregunta ¿cómo podemos dar cuenta de las razones de las acciones humanas? Y si es que, se ha desarrollado una teoría que ayude a dar cuenta de por qué actuamos como lo hacemos. Por lo que, para responder a esta cuestión, recurrí a la teoría de la explicación causal; esta teoría nos dice que las razones pueden ser las causas de las acciones, por lo que para poder argumentar en favor de esta teoría hay algunos puntos a analizar y explicar con el fin de que se pueda argumentar en su favor.

En primer lugar, se analizará el concepto de acción para poder sentar las bases de eso a lo que le podemos llamar así, y cómo es posible distinguirla de los meros sucesos que le pasan a la gente, porque no a todo lo que ocurre podemos llamarle acción ni por el simple hecho de poner un indicador gramatical, como el pronombre yo; cualquier suceso se puede llamar acción, dado que, por ejemplo, hay algunos movimientos que están programados en el cuerpo y no necesariamente son acciones, así que es importante dejar en claro este punto.

Intuitivamente diríamos que un sujeto se ve involucrado en las acciones para que podamos decir que hizo una acción y que los eventos son aquellos que ocurren fuera de un sujeto. Para poder hacer la distinción de lo anterior, daré una serie de ejemplos de unas y otros, para saber cuándo es una acción y cuándo no.

Analizaré el concepto de acción desde el punto de vista de Donald Davidson, dado que será uno de los principales autores que retomaré para mencionar los argumentos en favor de la teoría de la explicación causal, ya que desarrolla puntos importantes para la defensa de esta teoría. Para él las acciones tienen de fondo intencionalidad y estados mentales en los que se encuentra un sujeto para que pueda llevar a cabo una acción. Por lo que mencionaré 
cómo es que para Davidson se dan acciones intencionales, así como los efectos que en ocasiones se siguen de éstas.

\section{Análisis del concepto acción: distinción entre una acción $y$ un mero evento}

Para iniciar con este análisis es necesario sentar las bases sobre la diferencia entre una acción y un mero evento, me apoyaré en ejemplos para los argumentos, así la distinción será más precisa. También mencionaré algunas preguntas importantes de las que parto en esta investigación y trataré de responderlas de manera satisfactoria.

Podemos decir, intuitivamente y como comentario inicial sobre el análisis de las acciones, que una acción tiene una intención de fondo. La única razón por la que hacemos ciertas cosas, como apuntar, desplazarnos, abrazar, o cosas más específicas, como ir al refrigerador en el medio de la noche a tomar algo porque tenemos sed o que alguien dispare un arma y mate a una persona, es porque tienen una intención de fondo. No obstante, no podemos quedarnos con esa concepción sobre las acciones, dado que surgen preguntas acerca de la naturaleza de las acciones como, por ejemplo: ¿qué distingue una acción de un mero suceso?," ¿qué es lo

1 Davidson propuso un criterio para identificar los eventos - por tanto acciones- de manera causal. "Si e y $f$ son eventos simbólicos (o acciones), entonces $e$ es idéntica a $f$ si y sólo si e y $f$ tienen las mismas causas y efectos". Sin embargo este punto de vista es problemático porque es circular dado que es decir que $e$ y $f$ tienen las mismas causas y efectos, es decir que los mismos eventos causan a e y $f$ y los mismos eventos son causados por e y $f$.

El punto de vista de Jaegwon Kim es un tanto parecido al de Davidson, es decir, involucra tiempo, objetos y propiedades que determinan aún más la acción, "Si $a$ y $b$ son acciones simbólicas, entonces $a$ es idéntica a $b$ si y sólo si $a$ y $b$ son ejemplificaciones de la misma propiedad por el mismo objeto al mismo tiempo" (ver en O'Connor, Timothy, Sandis, Constantine, (eds.), A companion to the philosophy of action, Willey-Blackwell, United Kingdom, 2010, p. 18.). En un primer momento parece que el punto de vista de Kim muestra más ventajas ya que se puede decir que cierta propiedad no pudo haber sido ejemplificada por un objeto diferente, con una propiedad diferente en un tiempo diferente. 
que influye en la toma de decisiones sobre nuestro actuar, es decir, son razones internas (de estados metales) o razones externas (de creencias que tenemos acerca del mundo)?, ¿deberíamos pensar en las acciones como algo más que movimientos "generados por" algo?, ¿o pensar en las consecuencias de las acciones de manera física o mental?, o ¿qué teorías se han formulado para explicar las acciones? Todas estas preguntas se irán respondiendo, espero, de una manera satisfactoria a lo largo de este escrito; sin embargo, como primer paso comenzaré con las acciones, analizando el concepto, distinguiendo entre acciones genuinas y meros sucesos.

Ha habido preguntas acerca de la naturaleza de las acciones, es decir, en cuanto a cómo podemos distinguir de aquello que le pasa meramente a la gente y aquello que genuinamente hace. Cuando una persona va caminando y se tropieza o cuando le dan un golpe a alguien con una pelota son ejemplos de aquello que le pasa meramente a la gente, no hay una intención de fondo, ni un fin, sólo pasan y el agente que las experimenta no tiene ningún control sobre aquello que le ocurre. Por otro lado, está aquello que genuinamente hace la gente, como apuntar, abrazar, desplazarse, ente otras cosas; a esto le podemos dar el nombre de acciones, aunque es cierto que hay elementos que se involucran que hacen el análisis de las acciones más complejo que sólo dar una lista de las cosas que hacemos de manera consciente.

Si una persona mueve la cabeza, se desplaza de un lugar a otro o asiente con la cabeza, nos encontramos con una descripción de movimientos activos y pasivos. En A companion to the philosophy of action de Timothy O'Connor y Constantine Sandis, la diferencia entre los estados pasivos y los activos es que unos son los que están programados en nuestro sistema, por ejemplo: respirar, parpadear y básicamente todos los movimientos involuntarios que hacemos, de los cuales no nos damos cuenta que pasan, como el latir del corazón (que es un movimiento mecánico que nos mantiene con vida) o cuando una persona estornuda, tose, se sonroja o mueve las piernas, etcétera; éstas son cosas que un agente ha hecho, es decir, que las hace inconscientemente ya que una persona se encuentra en un estado pasivo mientras las realiza; pero las acciones huma-

Por ejemplo, John le disparó a Mary, ese evento fue, de hecho, una de las causas de la muerte de Mary que pudo haber sido causado por, digamos, que Peter le haya disparado a Mary exactamente de la misma manera, al mismo tiempo. 
nas no se reducen a esos ejemplos, sino que tienen una estructura psicológica más compleja.

Por su parte, los movimientos activos son aquellos que realizamos con más consciencia o que tienen una intención de fondo, es decir, como el desplazamiento de un lugar a otro o el mover algún miembro del cuerpo con el fin de hacer alguna actividad. Por ejemplo, cuando una persona mueve la cabeza ya sea en señal de estar de acuerdo con algo o porque tiene un mosquito en el oído y se mueve con el propósito de quitárselo de encima.

Davidson describe un ejemplo bastante ilustrativo en el que menciona cuáles son acciones y otras meras cosas que pudo haberle pasado (por lo que da a conocer una serie de sucesos en primera persona):

Esta mañana me despertó el sonido de alguien practicando el violín. Dormité un rato, luego me levanté, me lavé, me rasuré, me vestí y bajé las escaleras, y apagué, de paso, la luz del corredor. Me serví un poco de café, me tropecé con el borde de la alfombra del comedor y derramé el café cuando buscaba el New York Times ${ }^{2}$.

Entonces, Davidson se pregunta cuáles fueron meramente las acciones que él hizo y cuáles meros sucesos que le sucedieron. En primer lugar tenemos aquello que él hizo: levantarse, lavarse, rasurarse, bajar las escaleras y derramar el café. En segundo lugar, las cosas que sucedieron: su ser despertado, su tropezar con el borde de la alfombra. Y así, la lista podría extenderse si se detalla más la historia.

No obstante, muchas veces las propiedades y la manera en que respondemos tienen que ver con la forma en que se pregunta, o sea, cuando decimos que nos despertamos es algo que hicimos pero no es una acción como tal. Davidson afirma que hay una subclase de sucesos que son llamados acciones; por lo que hay que aclarar esto, al despejar algunas ideas erróneas sobre lo que regularmente solemos pensar que son las acciones.

Frecuentemente asumimos - de manera errónea- que al incluir un indicador gramatical para reconocer una acción ya es una acción como tal; por ejemplo: yo bailé, yo fui al parque, yo parpadeé,

2 Davidson, Donald, Ensayos sobre acciones y sucesos, Instituto de Investigaciones Filosóficas, UNAM, CRÍTICA, México, 1995, p. 63. 
yo me rasqué la cabeza, yo caminé tres kilómetros para ir a verte, entre muchos otros en los que el indicador con el que nos encontramos es el pronombre singular en primera persona: yo, seguido de verbos que parecen relacionarse con acciones que puede hacer un sujeto con algún fin u objeto.

Aunque es cierto que muchos verbos nos llevan a ese error porque decir que acusé, insulté, entre otros, nos posiciona directamente como autores de esos sucesos; no obstante, "mencionar a alguien en la posición de sujeto de una oración con el verbo en voz pasiva es, hasta donde puedo ver, asegurar que no es el agente de la acción. Pero, con mucha frecuencia, una oración registrará un episodio en la vida de un agente y nos dejará a oscuras acerca de si fue una acción" ${ }^{3}$. Que parpadeemos, rodemos en el pasto, tosamos, brinquemos, veamos la televisión, etcétera, en un primer momento parece que son acciones, pero no los sabremos hasta que no conozcamos qué más factores o características se involucran en esos sucesos.

Dicho esto y si concedemos por un momento que los verbos describen acciones, entonces puede ser el caso que lo que distingue a estos verbos puede ser la intencionalidad (siguiendo a Davidson en esta idea): he ahí por qué a algunos verbos los vemos como acciones que hace un agente, $y$ otros como meramente descriptivos.

Una persona realiza una actividad que tiene un objetivo $y$ comúnmente éste es el que las personas toman como base para una evaluación práctica general de sus opciones y oportunidades, es decir, el agente es consciente inmediatamente que está realizando la actividad en cuestión y que la actividad está dirigida por él hacia tal final elegido.

Si vemos un caso en los animales, Harry Frankfurt ha dicho que el comportamiento intencional en los animales constituye un nivel bajo de una acción activa, por ejemplo, cuando una araña camina por la mesa, la araña misma controla el movimiento de sus patas y las dirige para que la lleven de un lugar a otro. Esos movimientos tienen una finalidad para la araña y ésta es una explicación teleológica de las acciones. Por otro lado, el movimiento de mis dedos tal vez tenga el propósito de abrir un dulce, estos ejemplos son acciones en un sentido débil o básico.

También involucra el concepto de libertad, en el que presupone que en torno a las acciones se involucra un deseo mediante

3 Ibidem p. 64. 
con el que el agente se identifica. Cuando éste realiza una acción es inmediatamente consciente de la actividad en cuestión y de que esta actividad está apuntada hacia tal-y-tal fin ${ }^{4}$. Conviene recordar que "algunos filósofos aseveran que los movimientos del cuerpo de un agente no son nunca acciones. Es sólo el movimiento directo del agente, digamos, su pierna lo que constituye una acción física; el movimiento de la pierna es meramente causado por y/o incorporado como parte del acto del movimiento" 5 .

Ahora bien, hay cabida para preguntar si es que las contracciones de los músculos y en general todos los movimientos del cuerpo son acciones, mientras que el encender la luz y que la habitación se ilumine son meros resultados que suceden fuera del agente o que éste mismo causa, por lo que surge una duda: ¿cómo podemos distinguir entre dónde inicia una acción y dónde termina, es decir, si el resultado es la acción como tal, ya sea dentro del cuerpo del agente (como un estado mental) o en algún punto en la superficie del cuerpo (como si al hecho de mover la pierna para patear algo se le considera como una acción)?

Para responder a esa cuestión, consideremos que los estados mentales traen como consecuencia estados físicos. Un ejemplo clarificará este argumento: si tenemos sed, entonces debemos dirigirnos al refrigerador para saciar nuestra sed, por lo que el estado mental es saciar la sed y como resultado tenemos que vamos al refrigerador para saciar nuestra sed. Podemos clasificar esto como un evento o suceso físico.

En suma, el propósito del análisis del concepto acción era para distinguir entre aquello que es una mera acción y aquello que meramente le pasa a la gente. Por lo que tenemos como resultado que las acciones como tales son aquellas sobre las que una persona es consciente que hace y tienden a un fin o un propósito, y por otro lado están los eventos que meramente le pasan a la gente, éstos son aquellos que no tienden a un fin y suceden "fuera del sujeto", pero más que nada son aquellos sobre los que una persona no tiene control.

En las acciones -al ser sucesos sobre los que el sujeto ejerce control- hay intencionalidad, que es como un motivante a hacer algo. Una vez realizada la distinción entre acciones y eventos, es necesario analizar un punto de vista más concreto, el de Donald

4 Ibidem p. 2.

5 Ibidem p. 4. 
Davidson, quien describe las acciones y va más allá de sólo esta distinción, pues involucra el concepto de intención, por lo que a continuación presentaré sus argumentos.

\section{El concepto de acción en Donald Davidson}

Donald Davidson ha sido uno de los exponentes más importantes de la acción en filosofía, ya que sus ideas siguen siendo estudiadas, analizadas o refutadas, por lo que a continuación hablaré de su concepción acerca de las acciones. Éste nos dice, en un principio, que una acción es algo que hace un agente y que parece, si no es que lo es, intencional bajo alguna descripción. ${ }^{6}$ Afirma que hay una conexión importante entre una acción genuina, por un lado, y una intención, por el otro. Aunque claro está que deberíamos poder explicar tal conexión, se presentan varios problemas para realizarlo, como el hecho de que no hay una línea como tal en la distinción entre intenciones a futuro, actuar intencionalmente y actuar con cierta intención; por otro lado se encuentra el hecho de que el comportamiento humano es, en ocasiones, intencional bajo cierta descripción, pero no bajo otra. ${ }^{7}$

6 Ibidem p. 1.

7 Por ejemplo, Davidson dice que una persona puede provocarse a sí misma, intencionalmente, tropezar, pudo haber sido intencional bajo algún tipo de circunstancia, pero bueno, esto es sospechoso dado que, al parecer, nadie se causa a sí mismo tropezar, ya que sí nos podemos tropezar pero porque no nos fijamos qué es lo que hay en el piso o porque alguien nos empujó, pero nunca es intencional. (Sin embargo, este tema se analizará más a fondo en los siguientes apartados.) Parece que hay problemas con esta posición ya que para los conceptos de intención, intenciones hacia el futuro, actuar intencionalmente o actuar con cierta intención no se ha podido trazar la línea que los separe. Sobre la noción de que el comportamiento humano es a menudo intencional bajo alguna descripción, pero no bajo otra, Davidson pone el ejemplo de cuando una persona se causa a sí misma un tropiezo, la intención de esa persona bajo esa circunstancia es intencional mientras que, presumiblemente, la prevista pero involuntaria reacción del tropiezo que se causó no se supone que sea intencional bajo ningún concepto. Sin embargo, para decir que la acción fue intencional, que el agente se causó sí mismo el tropezar, se necesita que el tropiezo mismo y su causa activa estén presentes. 
He mencionado ya algunos ejemplos, de manera general, sobre lo que se dice en torno a las acciones. En la Stanford Encyclopedia of philosophy, mencionan un ejemplo destacado en Davidson: alguien alerta de la presencia de un ladón al iluminar la habitación, esto pasa al encender la luz, lo cual ocurre al presionar el interruptor (el encender la luz). Ahora bien, de acuerdo a la tesis de Davidson/Anscombe se dan cuatro eventos: "el alertar sobre el ladrón = el iluminar la habitación = el encender la luz $=$ el presionar el interruptor. $\mathrm{Y}$ esto es así a pesar del hecho de que el alertar sobre el ladrón fue no-intencional, mientras que el haber encendido la luz y que la habitación se iluminara desde un inicio sí fue intencional"

Pero muchas veces nos preguntamos cómo es que los estados mentales tienen efectos de manera física, para esto Davidson analiza la relación entre las razones y las acciones por lo que en el libro Ensayos sobre acciones y causas (específicamente en el primer ensayo), comienza con una pregunta sobre cómo es que se da la relación entre la razón y la acción cuando las razones son las que explican las acciones y ofrece una razón del agente para hacer lo que hizo, para lo cual responde que son las racionalizaciones. Asevera que la razón racionaliza la acción y explica que la racionalización es una especie de explicación causal, conforme a lo siguiente:

Una razón racionaliza una acción sólo si nos lleva a ver algo que el agente vio, o pensó ver, en su acción; algún rasgo, consecuencia o aspecto de la acción que el agente quiso, deseó, apreció; que le pareció atractivo, benéfico, obligatorio, agradable, o que consideró como su deber. No podemos explicar por qué alguien hizo lo que hizo diciendo simplemente que esa acción particular le pareció atractiva: tenemos que señalar qué fue lo que le pareció atractivo de la acción. Por lo tanto, siempre que alguien hace algo por una razón, puede caracterizársele: (a) como si tuviera algún tipo de actitud favorable hacia acciones de una clase determinada, y, (b) como si creyera (o supiera, percibiera, notara, recordara) que su acción es de esa clase. Deben incluirse en (a) actitudes tales como deseos, impulsos, instintos y una gran variedad de convicciones morales, principios estéticos, prejuicios económicos, convencionalismos sociales, metas y valores públicos y privados, en la medida

8 Idem. 
en que éstos puedan interpretarse como actitudes del agente dirigidas a cierta clase de acciones. ${ }^{9}$

Un aspecto importante, en este primer capítulo de Davidson, es que toma la actitud no sólo como los rasgos permanentes del carácter que se ven reflejados en la conducta de la gente, sino también como aquello que nos impulsa a una acción. "A menudo, dar la razón por la cual un agente hizo algo consiste en nombrar la actitud favorable (a) o la creencia relacionada (b) o ambas; permítaseme llamar a este par la razón primaria por la que el agente realizó la acción" ${ }^{10}$. Entonces, Davidson propone reformular el argumento de que las racionalizaciones son explicaciones causales y ofrece dos argumentos en favor de las razones primarias:

- Para entender cómo una razón de cualquier tipo racionali$z a$ una acción, es necesario y suficiente que veamos, por lo menos en sus rasgos esenciales, cómo construir una razón primaria.

- La razón primaria de una acción es su causa ${ }^{11}$.

El autor ofrecerá argumentos en favor de estas dos tesis recurriendo a un ejemplo ya mencionado: se trata del ladrón que es sorprendido cuando una persona se da cuenta de su presencia al oprimir el interruptor de la luz y encenderla. Ahora bien, Davidson afirma que al realizar este acto, que en realidad fue sólo uno, desató (por decirle de alguna manera) que ocurrieran cuatro descripciones. "Presioné el interruptor porque quería encender la luz, y al decir que quería encender la luz explico (doy mi razón, racionalizo) el acto de presionar el interruptor" ${ }^{\prime 2}$. Y no por esto se está racionalizando el hecho de que se haya alertado sobre el ladrón, ni que se haya encendido la habitación, ya que en primera instancia lo que quería hacer era presionar el interruptor, y dado este evento, surgieron como consecuencia: el alertar sobre el ladrón y que la habitación se iluminara. Estas dos consecuencias son cuasi intencionales, dado que se derivaron de una acción intencional.

9 Ibidem pp. 17-18.

10 Ibidem p. 18.

11 Idem.

12 Ibidem p. 19. 
Davidson menciona una condición necesaria para dar más precisión a los argumentos de las razones primarias:

$R$ es una razón primaria por la que un agente realizó la acción $A$ en la descripción $d$, sólo si $R$ consiste en una actitud favorable del agente hacia las acciones que poseen cierta propiedad y en una creencia suya de que $A$ en la descripción $d$ tiene esa propiedad $^{13}$.

Aunque la mayoría de las veces que describimos la razón primaria para una acción es correcta y la asumimos como verdadera, se dan casos en los que asumimos erróneamente la razón primaria; en éstos se ven involucradas las creencias y los deseos. Davidson lo expresa de la siguiente manera: "A menudo el querer y el desear se dirigen a objetos físicos. Sin embargo, 'quiero ese reloj de oro que está en la vitrina' no es una razón primaria y sólo porque sugiere una razón primaria explica por qué entré en la tienda: por ejemplo, sugiere que quería comprar el reloj" ${ }^{\prime 4}$.

Davidson considera que en ocasiones no es necesario mencionar la razón primaria de las acciones, dado que a veces el decir cuál es la consecuencia de hacer algo nos da o nos permite saber automáticamente cuál es la razón primaria, lo que expresa de esta manera:

Una razón primaria consiste en una creencia y en una actitud, pero generalmente es ocioso mencionar las dos. Si me dices que estás soltando el foque porque piensas que eso evitará que se vaya hacia atrás la vela mayor, no necesito que me digas además que quieres detener el desplazamiento hacia atrás de la vela mayor, y si dices que me estás haciendo una seña con los dedos para insultarme, no tiene objeto añadir que piensas que al hacerme esa seña con los dedos vas a insultarme. De manera semejante, muchas explicaciones de las acciones en términos de razones que no son primarias no requieren que se mencione la razón primaria para completarlas. Si digo que arranco las malas yerbas porque quiero que el césped esté bonito, sería fatuo añadir lo siguiente: 'Y, por tanto, considero que hay algo deseable en

13 Ibid, p. 20.

14 Idem. 
cualquier acción que embellezca el césped o tenga buenas probabilidades de embellecerlo'. ¿Por qué insistir en que hay algún paso lógico o psicológico, cuando se transfiere el deseo de un fin que no es una acción a las acciones consideradas como medios? Apoya igualmente el razonamiento que el fin deseado explica la acción sólo si el agente desea lo que concibe como medio ${ }^{15}$.

A continuación, Davidson asienta que no le parece necesario analizar y poner en cajas todas y cada una de las emociones humanas, sentimientos y demás, que puedan responder a la pregunta por qué lo biciste para conocer la razón primaria cuando nuestras expresiones son racionalizadas, porque asumimos que cuando damos una razón de este tipo es porque estamos respondiendo a una pregunta de tipo por qué.

Aunque es cierto que no siempre somos capaces de conocer del todo las razones primarias de nuestras acciones, Davidson afirma que siempre hay al menos una razón, dado que, por ejemplo, si sabemos que un hombre es claustrofóbico, está en una fiesta y lo vemos marcharse, podemos deducir que la razón primaria de su partida fue su claustrofobia y que no se sentía cómodo entre una multitud de personas. $\mathrm{O}$ cuando un hombre siente celos y éstos son el motivo de que haya un envenenamiento, dado que piensa que su acción va a dañar a un tercero, va a evitar el sufrimiento de algún otro, entre otras cosas. Por otro lado, tenemos casos en los que las razones primarias no nos parecen claras, sin embargo siempre existen.

La intencionalidad es un tema que comienza a entremezclarse con las razones primarias, pero en este caso el hecho de conocer la intencionalidad en las acciones no necesariamente nos otorga las razones primarias. Por ejemplo, si un chico va a la iglesia con la intención de complacer a su madre, entonces tiene una actitud favorable al agrado de su madre hacia él, pero es necesario conocer más información para saber si realmente la razón de por qué acude a la iglesia es porque quiere obtener el agrado de su madre o porque cree que es lo correcto, su deber o por alguna otra. Por eso, explicar el resultado de la acción que se pretende resulta muchas veces mejor que simplemente explicar aquello que era, o es, objeto de una intención o creencia.

15 Ibidem p. 21. 
Cada vez que hacemos algo por alguna razón -aunque en un sentido mínimo- nuestras racionalizaciones justifican las cosas que hicimos; Davidson lo plantea así: "desde el punto de vista del agente en el momento en el que actuó, había algo que decir a favor de la acción" ${ }^{\prime 16}$. Dado que las razones primarias, en cierto sentido, podemos tomarlas como razones base para lo que hacemos, de cierta manera podemos verlas como coherentes a como actuamos, aunque las razones sean efímeras o duraderas. Lo que estamos haciendo es ser animales racionales.

\section{Conclusión}

Para finalizar, tenemos que las razones racionalizan las acciones y de cierta manera éstas son las que hacen que una persona haga algo. Davidson asevera que hay ciertas actitudes favorables que un agente considera sobre sus creencias que hacen que haga algo; esas razones que se racionalizan las llama razones primarias, es decir, cuando alguien hace algo y decimos las razones de por qué hizo lo que hizo, hay ocasiones en las que nos es difícil identificar cuál es la razón primaria de una acción; por ejemplo cuando una persona presiona el interruptor, pero al presionar el interruptor enciende la luz y se da cuenta de que había un ladrón, en este caso el descubrir al ladrón no era la razón primaria sino que fue -por así decirlosecundaria. Una vez dicho esto, la intencionalidad se ve involucrada en las descripciones de Davidson, que es un tanto parecido a lo que mencionaba en el primer apartado, donde se refiere a que cuando hay intencionalidad en las acciones somos conscientes de lo que hacemos y actuamos en favor de esas razones.

16 Ibidem p. 24. 
